\title{
Trial of Functionalization of Silicon Tip Surface by Si-C Bonding
}

\author{
Tetsu Yonezawa, ${ }^{1}$ Naoto Shirahata, ${ }^{2}$ and Yoshiko Miura ${ }^{3}$ \\ ${ }^{1}$ Department of Chemistry, Graduate School of Science, The University of Tokyo, \\ Hongo, Bunkyo-ku, Tokyo 113-0033, Japan \\ Fax: 81-3-5841-2356, e-mail: yonezawa@chem.s.u-tokyo.ac.jp \\ ${ }^{2}$ National Institute for Materials Science (NIMS), 1-2-1 Sengen, Tsukuba, Ibaraki 305-0047, Japan \\ Fax: 81-29-860-4706, e-mail: shirahata.naoto@nims.go.jp \\ ${ }^{3}$ School of Materials Science, Japan Advanced Institute for Science and Technology (JAIST), \\ 1-1 Asahidai, Nomi, Ishikawa 923-1292, Japan \\ Fax: 81-761-51-1645, e-mail: miuray@jaist.ac.jp
}

Functionalization of silicon tip was carried out by photochemical/thermal hydrosilylation with an alkene-terminated sugar derivative. Si-C bond formation was suitable for preparation of monolayer on silicon surface. The tip was heated in a toluene solution of a sugar derivative with a special flask for hydrosilylation of small materials. With the functionalized tip, patterned surface of a silicon substrate was scanned.

Key words: SPM, silicon, alkene, hydrosilylation, sugar

\section{INTRODUCTION}

Functionalizing the metal and semiconductor surface is one of the hot research topics in chemistry, physics, materials science and modern nanotechnology [1-3]. There are many suitable organic molecules for this purpose. For example, organosilanes are good candidates for preparing an ordered monolayer on $\mathrm{SiO}_{\mathrm{x}}$ surface. On the other hand, hydrosilylation of hydrogen-terminated silicon to form $\mathrm{Si}-\mathrm{C}$ covalent bonds with alkenes or alkynes is also a very useful process to functionalize surfaces $[4,5]$. Hydrosilylation process which terminates the non-oxidized silicon surface with alkyl chains has made such active surface controllable under ambient conditions. Moreover, this process becomes a powerful tool to functionalize the bare silicon surface.

Carbohydrates form the major constituents of living organisms and work for cell differentiation, immune response, infection from viruses, as well as cancer metastasis. Carbohydrate molecules can have strong binding-affinity to specific proteins. Various carbohydrate arrays are commercially available, which are usually fabricated by ink-jet processes. On the other hand, monolayer-techniques have also been used to prepare such bio-sensing tools. Molecular orientation

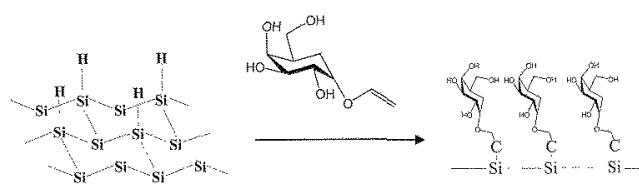

Fig. 1. Preparation of allyl-Gal SAM on hydrogen terminated Si surface. can be controlled based on the ordered structure formed by self-assembly processes. Thiols on gold are good candidates and various systems have already been proposed for bio-sensing. For example, carbohydrateprotein specific interaction can be detected by RAIRS (reflection absorption infrared spectroscopy) and SPR (surface plasmon resonance) [6] and QCM (quartz crystal microbalance) systems also useful for such specific sensing.

Recently, our group has proposed to use hydrosilylation to form carbohydrate monolayers immobilized $\mathrm{Si}-\mathrm{C}$ links on hydrogen terminated $\mathrm{Si}$ $(\mathrm{Si}-\mathrm{H})$ surface $[4,5]$. Bare-silicon surfaces have atomic flatness, chemical stability, reproducibility and surface homogeneity. Easy wet processes with $\mathrm{HF}$ and $\mathrm{NH}_{4} \mathrm{~F}$ with commercially available silicon wafers give such homogeneous surfaces. Therefore, silicon is highly suitable as the substrate for well-controlled monolayers.

Scanning probe microscopy (SPM) has been a powerful tool to study the surface properties because of its high spatial resolution. Chemical modification of SPM tips can enable a specific sensing with extremely high resolution.

In this study, immobilization of the carbohydrate molecules on silicon SPM probes was essayed by using photochemical/thermal hydrosilylation processes. Preparation and preliminary scanning images will be reported.

\section{EXPERIMENTAL}

Reagents were used as received. Water was purified by $\mathrm{Mili}-\mathrm{Q}$ system $(>18 \mathrm{M} \Omega$ ). As the functionalizing molecule on silicon tip surface, we have selected 1-allyl- $\alpha$-D-galactopyranoside (Allyl-Gal), which was synthesized as previously reported [7]. This type of molecules can homogeneously cover the hydrogen-terminated silicon surface by photo-assisted hydrosilylation or thermal hydrosilylation [5]. A UV lamp (San-ei Electronics, Japan, UVF-203S) was used 


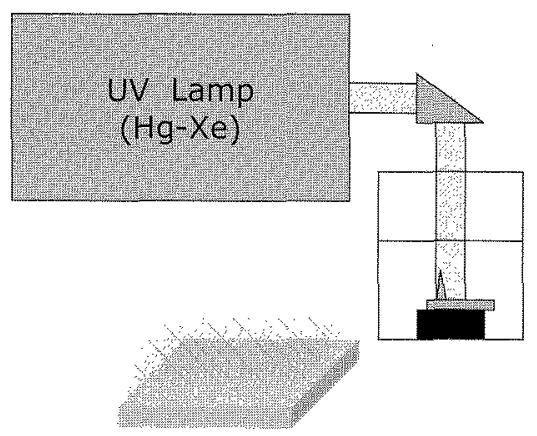

Fig 2. Illustration of preparation of modified probes.

for hydrosilylation. As Si SPM tips, SI-AF01 (Seiko Instrument) was used. In this work, thermal hydrosilylation process was also used to functionalize Si tips. The contamination on the surface of Si probes was eliminated by irradiating an eximer lamp. The surface of $\mathrm{Si}$ was oxidized to $\mathrm{SiO}_{2}$. After cleaning by $\mathrm{UV} / \mathrm{O}_{3}$, the probe was treated by HFaq. Modification of Si probes was carried out by a photo-hydrosilylation procedure. The modified tips were used to scan the modified silicon surface generated by photo-lithography and hydrosilylation. Seiko Instrument system was used for surface analysis under ambient atomosphere. Modification of silicon surface was carried out by using a photo-assisted hydrosilylation process. SPM probes were first immersed into the solution of Allyl-Gal. Then, UV lamp was irradiated from upside.

For the samples, photo-patterned Si wafers were used [8]. An alkyl-monolayer covered $\mathrm{Si}$ wafer was photo-patterned by UV-irradiation through a TEM grid as the template. The UV-irradiated area was turned into $\mathrm{SiO}_{\mathrm{x}}$ which is hydrophilic and the non-irradiated area was remaining hydrophobic.

\section{RESULTS AND DISUCSSION}

Recently, surface modification of SPM probes has been reported in order to specify the finding molecules and/or electronic states of the surface. Monolayer systems can be used as the most effective way to modify the surface of SPM probes $[9,10]$ In many cases, gold-coated tips are used for the surface modification and thiol compounds are attached to the gold-coated surface because Au-S interaction is enough strong and stable. Furthermore, gold is precious and inert material which can be readily deposited on a small surface area by sputtering as well as by evaporation. Simple immersing cleaned SPM probes into a thiol compound solution gives a uniform SAM layer on the gold surface. Such probe modification may control the electron tunneling between the probe and the sample surface. Nishino et al. have reported 4-mercaptobensoic acid modified gold-covered probes [10].

Modification of Si type SPM probes can be one of interesting steps to functionalize SPM probes for various studies. Modification of scanning probes was carried out by using hydrosilylation after HF treatment in order to remove native oxidized surface $\left(\mathrm{SiO}_{x}\right)$. The probes were introduced into a separable flask with a reaction stage in it (Figure 2). UV-irradiation was carried out thorough a quartz plate put on a glass vessel in order to avoid the

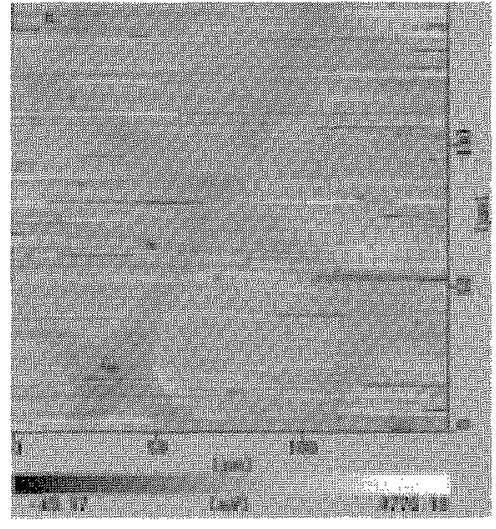

Fig. 3. Lateral force microscopic (LFM) image of $\mathrm{SiO}_{\mathrm{x}} /$ alkyl pattern on $\mathrm{Si}$ scanned by a sugar modified probe.

evaporation of the solvent. The modified SPM probe was washed and dried before usage.

Figure 3 shows the LFM scanning image of the photo-patterned silicon surface. As shown in this image, $\mathrm{SiO}_{x}$ area was brightly indicated probably due to the stronger interaction between $\mathrm{OH}$ groups of Gal parts and the $\mathrm{SiO}_{\mathrm{x}}$ surface. LFM images can be obtained several times with one modified probe, but water molecules adsorbed on tip affect the quality of the image. The minimum lateral resolution of the modified probe has not been determined yet. It should be also affected by the adsorbed water molecules on sugar groups, and in order to obtain the minimum resolution, observation under vacuum condition should be indispensable. Detailed characterization of the surface of the modified probes and their application will be examined in the near future.

Acknowledgement

This work is supported by The Kurata Memorial Hitachi Science and Technology Foundation. TY thanks Prof. H. Nishihara (Univ. Tokyo) for allowing him to carry out this work. Authors thank Dr. S. Furumi of NIMS for his experimental assistance on a UV-irradiation process.

References

[1] A. Ulman, Chem. Rev., 96, 1533 (1996).

[2] M. Ara, H. Graaf, and H. Tada, Jpn. J. Appl. Phys., 41, 4894 (2002).

[3] H. Sato, Y. Miura, T. Yamauchi, K. Kobayashi, N. Saito, O. Takai, Trans. Mater. Res. Soc. Jpn., 31, 659 (2006).

[4] J. M. Buriak, Chem. Rev, 102, 1271 (2002).

[5] N. Shirahata, A. Hozumi, and T. Yonezawa, Chem Rec., 5, 145 (2005).

[6] D. J. Revell, J. R. Knight, D. J. Blyth, A. H. Haines, D. A. Russell, Langmuir, 14, 4517 (1998).

[7] N. Shirahata, T. Yonezawa, Y. Miura, K. Kobayashi, and K. Koumoto, Langmuir, 19, 9107 (2003).

[8] N. Shirahata, T. Yonezawa, W.-S. Seo, and K. Koumoto, Langmuir, 20, 1517 (2004).

[9] T. Nishino and Y. Umezawa, Sens, Lett., 3, 231 (2005).

[10] T. Nishino, P. Bühlmann, T. Ito, and Y. Umezawa, Surf. Sci., 490, L579 (2001) 